\title{
Can a synergistic activation of pattern recognition receptors by plant immunomodulators enhance the effect of oncologic therapy? Case Report of a patient with uterus and ovary
} sarcoma

\author{
Tibor Hajto ${ }^{1 *}$, Lilla Baranyai ${ }^{1}$, Angelika Kirsch ${ }^{2}$, Monika Kuzma $^{1}$ and Pal Perjési ${ }^{1}$ \\ ${ }^{1}$ Institute of Pharmaceutical Chemistry, Medical University Pecs, Hungary \\ ${ }^{2}$ Medical Office, Paradiestrasse 14, CH-4102 Binningen, Switzerland
}

\begin{abstract}
Background: Growing evidence supports the hypothesis that similar to microbes various plant extracts can also contain Pathogenic Associated Molecular Pattern (PAMP)-like structures which can activate type-1 cellular functions of the innate immune system. Since they are important in tumor defense and the chemical production of PAMP structures is hardly accomplishable, the plant extracts standardized concerning their PAMP like structures may be promising for future tumor therapy.
\end{abstract}

Method: The synergistic effect of two standardized plant immunomodulators was monitored by the hemocytological measurement of the peripheral level of Natural Killer (NK) cells. Suboptimal doses of mistletoe lectins (ML) and Arabinoxylan in MGN-3 were compared using healthy volunteers.

Results: $24 \mathrm{~h}$ after a single suboptimal dose $(15 \mathrm{mg} / \mathrm{kg})$ of Arabinoxylan in MGN-3 an average increase (+/-SEM) in NK level was 46.4 (+/-36)\% and 24h after a single suboptimal $(0.45 \mathrm{ng} / \mathrm{kg}) \mathrm{ML}$ injection a $36(+/-13) \%$ enhancement was found. If these suboptimal doses of Arabinoxylan and ML were given together, a highly significant enhancement $(293+/-41 \%)$ was established indicating a high significant synergism between them ( $<<0.001)$. A patient with uterus and ovary sarcoma was not able to tolerate the CYVADIC chemotherapy. After its combination with ML and Arabinoxylan using optimal doses: $0.75 \mathrm{ng} / \mathrm{kg}$ and $45 \mathrm{mg} / \mathrm{kg}$ respectively, she received six cycles CYVADIC and thereafter only immunotherapy was given. During the following five years she has regularly been tumor free.

Conclusion: The combination of standardized plant extracts with PAMP-like structures seems to open new perspectives in the supportive therapy of metastatic tumors. Further research is necessary.

\section{Introduction}

In many cases of malignant tumors with advanced stages of disease the conventional chemotherapy is not, or hardly tolerated, because of severe side effects. These patients are often in poor state of health, which can hinder the further treatment and worsened the prognosis of their disease. Several authors [1,2] suggest that in this intolerance to oncologic therapy the behavioral co-morbidities of tumor patients (such as fatique, depression, pains and cognitive dysfunction) can also play an important role. Growing evidence suggests that these behavioral symptoms in cancer patients are often associated with decreased activity of their cellular immune responses by the innate immune system with enhanced activity of regulatory $\mathrm{T}$ cells [1]. Therefore, it is noteworthy referring to previous case reports [3] of six patients with sarcoma, showing astonishing remission under an immunomodulatory treatment using plant extracts which were standardized as to their mistletoe lectin (ML) content. As reported previously [4,5], ML represents a Pathogenic Associated Molecular Pattern (PAMP) like structure originating from plant leaves and stem which can bind appropriate Pattern Recognition Receptor (gangliosides with terminal Neu5Ac alpha 2-6Gal beta $1-4$ GlcNAc residues) on membrane of the type-1 phagocytic cells (such as M1 macrophages or CD1a dentritic cells).As known, PAMP - PRR (in the case of MLit is a lectin-sugar) interaction on membrane of type-1 phagocytic cell generate proinflammatory cytokines and IL12 activating cytotoxic effector cells, such as Natural Killer (NK) and NK-T cells which are potent inhibitor of tumor growth [6-11]. Since NK cells are regularly present in peripheral blood, they are very suitable for monitoring the activation level of type- 1 cellular cascades in innate immune system. Consequently, measurements of NK responses in peripheral blood of patients render possible a better judgment of the efficacy of an immunomodulator. This possibility for monitoring the type-1 natural immune cells in blood encouraged to ask the question whether a combination of ML with another immunomodulator (showing similar PAMP like structures) can result in more benefit? In this paper using healthy volunteers the NK responses will be presented after a single application of mistletoe lectin (ML) and Arabinoxylan

Correspondence to: Tibor Hajto, Institute of Pharmaceutical Chemistry, Medical University Pecs, Meggyes u. 26, H-2045 Törökbálint, Hungary, Tel: +36 309735 337; E-mail: drhajtot@t-online.hu

Keywords: uterus and ovary sarcoma, immunomodulation, mistletoe lectin, arabinoxylan

Received: September 20, 2015; Accepted: October 20, 2015; Published: October 23,2015 
Hajto T (2015) Can a synergistic activation of pattern recognition receptors by plant immunomodulators enhance the effect of oncologic therapy? Case Report of a patient with uterus and ovary sarcoma

given in standardized plant preparations. In addition, a case report of a patient with uterus and ovary sarcoma will be presented using this combination.

\section{Materialand methods}

\section{Mistletoe extract standardized by the determination of its mistletoe lectin level}

Iscador $^{R}$ is a fermented aqueous mistletoe plant extract manufactured and supplied by Weleda AG (CH-4144 Arlesheim, Switzerland). The active (sugar-binding) lectin content of commercially available mistletoe extracts (Iscador ${ }^{R} \mathrm{M}$ spec $5 \mathrm{mg}$ ) was measured in the research laboratory of Pharmceutical Chemistry Department of Medical University Pécs.

The determination of sugar binding mistletoe lectin (ML) level in extracts was carried out by an optimized ELLA technique as published previously [4]. Standardized ME exhibited a bell-shaped dose-response relationship and $0.5-1.0 \mathrm{ng} / \mathrm{kg}$ lectin doses were found to be most effective as it was always assessed previously using healthy volunteers. Since two and three therapy-free days were found to be necessary for an immunologically optimal effect, the subcutaneous ME injections were regularly given twice a week.

\section{Standardized rice bran extract (BioBran/MGN-3)}

The second immunomodulatory drug used in the combinative treatment of the presented patients is BioBran/MGN-3 which is manufactured and supplied by Daiwa Pharmaceutical Co, Ltd, Tokyo, Japan. BioBran/MGN-3 is composed of denaturated hemicellulose, which is obtained by rice bran hemicellulose reacting with multiple carbohydrate-hydrolyzing enzymes from shiitake mushrooms. BioBran/MGN-3 is standardized for its main chemical component: arabinoxylan with a xylose (in its main chain) and with an arabinose polymer (in its side chain). To the presented patients BioBran/MGN-3 was given orally in doses between 12 and $45 \mathrm{mg} / \mathrm{kg}$ twice a week parallel to the optimized, lectin-oriented mistletoe extract therapy.

\section{Investigation of NKlevel in six healthy volunteers}

The frequency of Large Granular Lymphocytes (LGL) was tested by a cytological method used in hematology [12]. As reported previously, the majority of these cell populations are NK cells and their peripheral level correlate well with their cytotoxic activity [12]. Six healthy volunteers (females, average age 24.6 year) were tested for three consecutive weeks: after a single mistletoe extract injection, after a single MGN-3 therapy and after a combined application of both. The differences in relative augmentations were analyzed with Student's $t$ test and $\mathrm{U}$ test according to Wilcoxon, Mann and Whitney using the Statgraphics statistical package for IBM-compatible computers.

\section{Ethics committee}

The ethics committee proposed to observe and publish case reports of patients treated by standardized plant extracts. This case report and previous one may stimulate an interest for the other research groups according to the opinion of the ethics committee. The presented patient has given an informed consent to process and publish her dates. She was informed that plant extracts can be regarded as a complementary therapy and it cannot be a substitute for conventional oncologic treatments. The patient was regularly controlled in an oncologic center and simultaneously, she was also treated with plant immunomodulators and monitored.

\section{Results}

Since previous reports with plant lectin (ML) preparation alone or in combination with conventional oncologic therapy showed astonishing clinical results [3], the question has arisen whether the combination of various PAMP like structures with plant origin can improve the immunomodulatory effects of each other? Therefore the effect of ML and Arabinoxylan on peripheral level of NK cells was examined using six volunteers and as shown in Figure 1, a highly significant synergism was established. $24 \mathrm{~h}$ after a single suboptimal dose $(15 \mathrm{mg} / \mathrm{kg})$ of Arabinoxylan an average increase (+/-SEM) in NK level was $46.4(+/-36) \%$ and $24 \mathrm{~h}$ after a single suboptimal $(0.45 \mathrm{ng} / \mathrm{kg})$ ML injection a $36(+/-13) \%$ elevation was found. As shown in Figure 1, if these suboptimal doses of Arabinoxylan and ML were given together, a highly significant enhancement $(293+/-41 \%)$ was established indicating a high significant synergism between them $(\mathrm{p}<0.001)$.

In this paper a case report of a now 33 years old patient is presented who five years ago belonged to the first tumor patients treated regularly with the combination of optimal dose of ML $(0.75 \mathrm{ng} / \mathrm{kg})$ and with an optimal dose of arabinoxylan ( $45 \mathrm{mg} / \mathrm{kg})$ twice a week. In November 2010 the first diagnosis of left ovary sarcoma took place which was removed by surgery. Three weeks later a $52 \times 36 \mathrm{~mm}$ endometrium sarcoma with a metastasis in the right ovary was detected in CT and by biopsy (Figure 2A). A second surgery (hysterectomy and adnexectomy) was carried out. The histological diagnosis showed an undifferentiated endometrium sarcoma with metastasis in ovary. The Ca-125 was 114.8.

Four weeks after the second surgery an adjuvant chemotherapy with a CYVADIC protocol (Cyclophosphamide, Vincristine, Adriamycin and Dacarbazyne) was started. However, the patient had very severe side effects and therefore the third cycle of CYVADIC was given only after eight months. The quality of life was very bad. The patient was totally exhausted. She was regularly on the point of collapse. At the same time, with the third cycle (July 2011) the immunomodulatory treatment with $0.75 \mathrm{ng} / \mathrm{kg}$ lectin and $45 \mathrm{mg} / \mathrm{kg}$ Arabinoxylan was started and regularly given twice a week. After the start of the immunotherapy a rapid improvement of quality of life was observed. She was able to

\section{Synergic Effect of Mistletoe Lectin and} Arabinoxylan on Level of Circulating NK Cells

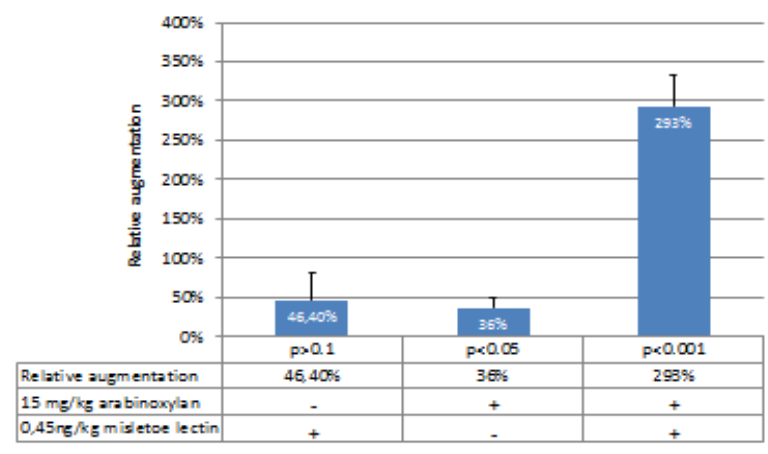

Figure 1. The peripheral level of Natural Killer (NK) cells was measured by a cytological method used in the hematology [12]. 24h after a single suboptimal dose $(15 \mathrm{mg} / \mathrm{kg})$ of Arabinoxylan the average increase (+/-SEM) in NK level was $46.4(+/-36) \%$ and $24 \mathrm{~h}$ after a single suboptimal $(0.45 \mathrm{ng} / \mathrm{kg}) \mathrm{ML}$ injection a $36(+/-13) \%$ elevation was found. If these suboptimal doses of arabinoxylan and ML were given together, a highly significant enhancement $(293+/-41 \%)$ was established indicating a highly significant synergism between them $(\mathrm{p}<0.001)$. 
A.)

\section{Uterus sacoma with metastasis in left ovary}

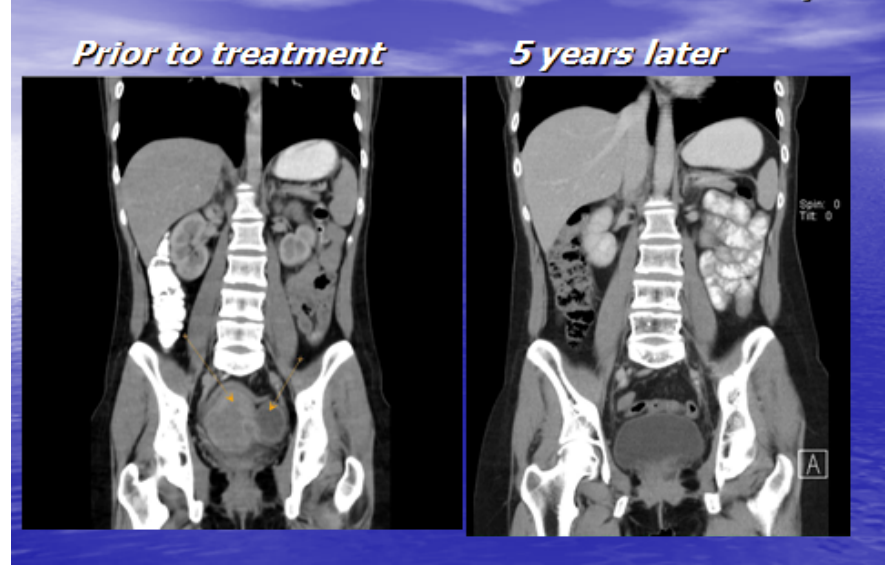

Figure 2. Case report of a patient with uterus and ovary sarcoma who was treated with adjuvant CYVADIC chemotherapy. Because of severe side effects the chemotherapy was only possible after its combination with immunomodulatory treatment of ML and Arabinoxyle preparations given in optimal doses $(75 \mathrm{ng} / \mathrm{kg}$ and $45 \mathrm{mg} / \mathrm{kg}$, respectively) twice a week. Figure $2 \mathrm{~A}$ shows the tumors prior to chirurgical removal (little arrows point to her tumors) and Figure 2B illustrate her CT control investigation after 5 years presenting that she is completely tumor free.

tolerate the next three cycles of CYVADIC always better. By now (after five years) she has regularly been checked by $\mathrm{CT}$ investigations and she has always been tumor free (Figure 2B). Ca-125 values are regularly normal (between 6 and 8). Her quality of life is excellent.

\section{Discussion}

According to the data of great oncologic centers the average survival of soft tissue sarcoma with stage IV of the disease after five years is less than 20\% [13]. Metastatic soft tissue sarcoma cases after CYVADIC chemotherapy retrospectively reviewed showes that the response rate was $33 \%$ and the median progression free survival was 7.4 months [14]. Therefore, the case report of the patient with advanced soft tissue sarcoma, presented in this paper, is surprising. The combination of CYVADIC with standardized plant immunomodulators rendered possible the application of the third, fourth, fifth and sixth cycle. After the chemotherapy she has received only immunomodulatory treatment. This permanent complete remission during five years without any further oncologic treatment suggests the hypothesis that a combination of CYVADIC chemotherapy with plant immunomodulators exhibiting a synergestic effect with each other, can improve the prognosis of patients with advanced soft tissue sarcoma.

It is now generally recognized that immunocompetent cells of the innate immune system are workingin two separate directions: 1. Prototypic M1 macrophages and CD1a+ dentritic cells (DC1) generating IL-12 and other pro-inflammatory cytokines activating cytotoxic effector cells such as natural killer (NK) cells. They are potent inhibitors of tumor growth. 2. Prototypic M2 macrophages and DC2 dentritic cells generating IL-4 and IL-10, which facilitate the generation of Th2 cells while inhibiting Th1 cells. They can induce inflammation and promote cell proliferation by producing growth factors and through initiation of the arginase pathway, neoangiogenesis and tissue repair [8]. In patients with malignant tumors a higher than normal proportion of macrophages belongs to this prototypic M2 population and this appears to alter the balance of immune responses [10]. For example, in healthy individuals M2 monocytes were found to comprise only $10 \%$ of the total monocytic population, and in tumor patients this proportion was $40 \%$ higher [6]. Since many years, a beneficial influence of certain bacterial infections on the progress of tumor disease has often been observed. Now, it is also clear that bacterial Pathogenic Associated Molecular Pattern (PAMP) molecules can stimulate the type 1 natural immune cells by binding the Pattern Recognition Receptors (PRR) on their membrane. However, in spite of the fact that the effects of a great number of bacterial preparations on tumor disease were investigated, there are not in clinical use because of toxic side effects. In case of endotoxin tolerance could be observed. Therefore the hypothesis is interesting that plants may also contain PAMP like molecules without toxic side effects. Plant immunomodulators used for the treatment of the sarcoma patients have no side effects and they combine with each type of oncotherapy as reported previously [15].

Since ML and arabinoxylan showed a highly significant synergism, the combination of various standardized plant immunomodulators containing PAMP like structures, may open new perspectives in the tumor therapy.

\section{Acknowledgments}

The authors thank Mrs. Cornelia van den Bergh (for her grants to support this research).

\section{Disclosure statement}

The authors declare that there is no competing or other conflicting interest in relation to this paper.

\section{References}

1. Liu WM, Meyer B, Dalgleish AG (2009) How immunotherapy can enhance the response to other modalities and improve outcome and quality of life. $J$ BUON 14 : S103-109. [Crossref]

2. Miller AH, Ancoli-Israel S, Bower JE, Capuron L, Irwin MR (2008) Neuroendocrineimmune mechanisms of behavioral comorbidities in patients with cancer. J Clin Oncol 26: 971-982. [Crossref]

3. Kirsch A, Hajto T (2011) Case reports of sarcoma patients with optimized lectinoriented mistletoe extract therapy. J Altern Complement Med 17: 973-979. [Crossref]

4. Hajtó T, Fodor K, Perjési P, Németh P (2011) Difficulties and perspectives of immunomodulatory therapy with mistletoe lectins and standardized mistletoe extracts in evidence-based medicine. Evid Based Complement Alternat Med: 298972. [Crossref]

5. Muthing J, Meisen I, Bulau P, Langer M, Witthohn K, et al. (2004) Mistletoe lectin I is a sialic acid-specific lectin with strict preference to gangliosides and glycoproteins with terminal Neu5Ac alpha 2-6Gal beta 1-4GlcNAc residues. Biochemistry 43: 29963007. [Crossref]

6. Nagtegaal ID, Klein-Kranenbarg E, Mulder-Stapel A, Hermans J, Van den Velde CJH, et al. (2001) Local and distant recurrences in rectal cancer patients are predicted by the nonspecific immune response; specific immune response has only a systemic effect. A histopathological and immunohistochemical study. BMC Cancer 1: 7-16. [Crossref]

7. Ostrand-Rosenberg S, Sinha P (2009) Myeloid-derived suppressor cells: linking inflammation and cancer. J Immunol 182: 4499-4506. [Crossref]

8. Mantovani A (2007) Inflammation and cancer: the macrophage connection. Medicina (Buenos Aires) 67 (Suppl II): 32-34.

9. Terabe M, Berzofsky JA (2008) The role of NKT cells in tumor immunity. Adv Cancer Res 101: 277-348. [Crossref]

10. Baskic D, Acimovic L, Samardzic G, Vujanovic NL, Arsenijevic NN (2001) Blood monocytes and tumor-associated macrophages in human cancer: differences in activation levels. Neoplasma 48: 169-174. [Crossref]

11. Sanchez-Torres C, Garcia-Romo GS, Cornejo-Cortes MA, et al. (2001) CD16+ and CD16- human blood monocyte subsets differentiate in vitro to dendritic cells with different abilities to stimulate CD4+ T cells. Int Immunol 13: 1571-81. [Crossref] 
Hajto T (2015) Can a synergistic activation of pattern recognition receptors by plant immunomodulators enhance the effect of oncologic therapy? Case Report of a patient with uterus and ovary sarcoma

12. Hajto T, Hostanska K, Weber K, Zinke H, Fischer J, et al. (1998) Effect of a recombinant lectin, Viscum album agglutinin (rVAA) on secretion of interleukin-12 in cultured human peripheral blood mononuclear cells and on NK-cell-mediated cytotoxicity of rat splenocytes in vitro and in vivo. Nat Immun 16: 34-46. [Crossref]

13. Singer S, Maki RG, O’ Sullivan B (2011) Soft tissue sarcoma. In: DeVita VT, Hellman S, Rosenberg SA (Eds.), Cancer: principles and practice of oncology. Philadelphia, Wolters Kluwer/Lippincott William@Wilkins p. 1550.
14. Nakano K, Motoi N, Takahashi S (2015) The CYVADIC regimen as a combination chemotherapy treatment option for advanced soft tissue sarcomas originating from the non-extremities. Intern Med 54: 187-93. [Crossref]

15. Hajto T, Kirsch A (2013) Case reports of cancer patients with hepatic metastases treated by standardized plant immunomodulatory preparations. J Cancer Res Update 2: 1-9.

Copyright: $\odot 2015$ Hajto T. This is an open-access article distributed under the terms of the Creative Commons Attribution License, which permits unrestricted use, distribution, and reproduction in any medium, provided the original author and source are credited. 